\title{
LXXXIII. On the conducting power of iodine for electricity
}

\section{James Inglis M.D.}

To cite this article: James Inglis M.D. (1836) LXXXIII. On the conducting power of iodine for electricity, Philosophical Magazine Series 3, 9:56, 450-452, DOI: 10.1080/14786443608649039

To link to this article: http://dx.doi.org/10.1080/14786443608649039

曲 Published online: 01 Jun 2009.

Submit your article to this journal $\pi$

Џll Article views: 2

Q View related articles $\sqsubset$ 
what would be in general an imaginary value of $a^{x}$, if $a$ and $x$ were real and positive. But I have already occupied too much space, and need not labour these arguments, for Professor De Morgan does not materially differ from me here. He seems to regard the ordinary theory as an edifice complete in itself, but is content to receive my results as an extension which may prove useful, whereas I regard them rather as the erection of a wing, required for symmetry, if not for use.

LXXXIII. On the Conducting Power of Iodine for Electricity. By James IngLis, M.D.*

[Addressed to the Chemical Section of the British Association.]

I $T$ may not, perhaps, have escaped the notice of some of the members of this Section, that in extracts from a Prize Essay of mine, published some months ago, in the Philosophical Magazine, I stated that I had found iodine to be a conductor of electricity. Nor may the experiments of $\mathrm{Mr}$. Solly tending to prove the contrary have passed by unobserved. Nevertheless, being satisfied in my own mind what I had published was correct, I determined at the earliest opportunity to resume the investigation, and instead of answering that gentleman directly through the medium of the Philosophical Magazine, I thought it might be better to lay before you the result, in as much as I shall by experiment prove my former statement, and then furnish you with that portion of iodine which you have seen conduct, that you may for yourselves judge of its purity.

In Mr. Solly's first paper, no mention is made of experiments performed with fused iodine; but his attention being drawn to the subject by a note of mine, he published a second, in which he throws a doubt on the purity of the iodine $I$ had used, saying that it contained " most probably the iodide of iron, which is not unfrequently present in the jodine of the shops." (Lond. and Edinb. Phil. Mag., No. 48. p. 401.)

The iodine I used was obtained from the manufactory of $\mathrm{Mr}$. Whitelaw of Glasgow, where no iron vessel is ever employed, and in which, in its veriest impurity, no iron can be detected. Here, for instance, is one tube containing an aqueous solution of ioduret of iron; a second, an aqueous solution of the iodine to be tested; and a third having in it a solution of the ferrocyanate of potassa. Now, on adding a small portion of this last solution to the one containing iron, immediately the blue ferrocyanate of the peroxide of iron results. But no such effect is produced when the test is added

* Read before the Chemical Section of the British Association at Bristol, Aug. 26, 1836: and now communicated by the Author. 
to the solution of iodine; it remains the same as before admixture. Add, however, but a single drop of the solution of the ioduret of iron and the blue colour instantly appears. But supposing that a small portion of the ioduret by some chance happened to be present, we know that from its great affinity for water it could be removed by washing; I therefore washed several times, and thoroughly dried, the iodine with blottingpaper; and lastly, thrice sublimed it; so that now I presume it is as pure as possible.

Of this iodine thus prepared, I put a portion into a tube with a platinum wire hermetically sealed into one extremity; and introducing a second wire at the other till one end approached the former to within about the fourth of an inch, I hermetically sealed this extremity also: so that we have here a closed tube containing dry pure iodine, with two separate platinum wires conmunicating together only through the medium of the iodine.

Three galvanic troughs, containing each 30 pairs of plates, were charged, (but 20 pairs, or fewer, as in the trough now to be used, are sufficient,) and one of the platinum wires fixed to the positive pole, whilst the other was placed in a glass of acidulated water. On forming the galvanic circle, no effect was produced, either by the decomposition of water, or by sensation on the tongue: nor was there any difference on reversing the poles.

The iodine being now liquefied by the flame of a spirit-lamp, and the tube attached to the negative pole, the platinum wire was placed as before in water; and on completing the circle by a copper wire from the positive, instantly bubbles of gas appeared and were evolved at the platinum wire, whilst none appeared at the copper, being positive. The order being reversed, globules of gas appeared at both wires, showing clearly that decomposition had been effected.

Again, if the platinum wire be placed on the tongue, and the copper wire be taken hold of with the hand, instantly the galvanic sensation is felt.

The heat being removed, the power of conduction gradually dies away; so that in seven minutes it is incapable of transmitting even sufficient electricity to be perceived by the tongue. When therefore I stated in a note attached to Mr. Solly's paper, that iodine when cold and concrete still conducted, I was in error, being led to say so from recollection only. But my general statement that iodine is a conductor, is, I hope, satisfactorily shown to be borne out this day by experiment.

Dr. Cumming considered that the conduction might be $3 \mathrm{H} 2$ 


\section{The Rev. J. W. MacGauley on some remarkable Results}

explained by the fact made known by Mr. Faraday, that air when heated becomes a conductor. But that could not apply here, for in the first place, it is not air at all that is the medium of conduction, it is liquid iodine; and in the second, on melting the iodine and inverting the tube the conduction is suspended.

Dr. Apjohn now suggested that the iodine at the temperature required for its liquefaction might act on the platinum, and that an ioduret of platinum thus formed would conduct.

But iodine does not act on platinum at $225^{\circ}$ Fahr., and $225^{\circ}$ is the point at which iodine fuses.

This I stated at the time the objection was made, and since my return $I$ have accurately weighed a piece of platinum wire, and allowed iodine to act on it for half an hour, at and above the point of fusion; when on weighing again, the platinum wire was found to have lost nothing ; so that Dr. Apjohn's objection thus loses its weight, no ioduret having been formed.

The conducting power of iodine, atmospheric air, and some other substances when heated, and their non-conducting when cold, adds, I think, an argument in favour of that theory which considers electricity to be but an action of matter; and heat and electricity to be but modifications of each other.

Castle Douglas, Oct. 3, 1836.

J MMES INGLIS.

LXXXIV. An Account and Explanation of some remarkable Results obtained during a Course of Electro-Magnetic Experiments. By the Rev.J. W. MACGAULEY*.

I $\mathrm{T}$ is impossible not to remark that the electro-magnetic helix seems to increase the power of a given battery, for the brilliancy of the spark increases with the magnitude of the apparatus. I expected that such an intensity might be given by a very powerful electro-magnet, as that a small galvanic arrangement and a single circle might be made to communicate a considerable shack. I coiled nearly 2000 feet of copper wire, in ten helices, upon a bar of soft iron, during the experiments I was making preparatory to the construction of a large and greatly improved machine on the principle which I exhibited last year (1835) to the British Association, and which is now nearly completed: from this magnet I obtained a powerful shock.

It is not necessary to detail a great variety of arrangements adopted and results obtained; among others I came to the following conclusions :

* Read before the Royal Dublin Society on June 14th; and now communicated by the Author. 